\title{
Using Zeolitic Core@Shell Adsorbents for the Selective Removal of Free Glycerol from Crude Biodiesel
}

\author{
Nima Masoumifard,${ }^{[a, b, d]}$ Pablo M. Arnal,${ }^{[c]}$ Serge Kaliaguine ${ }^{*[b]}$ and Freddy Kleitz ${ }^{*[a, d]}$
}

\begin{abstract}
Selective adsorption of free glycerol from crude biodiesel mixture has been investigated using mesoporous silica spheres coated with a thin shell of microporous silicalite-1. Various types of mesoporous silica spheres with different sizes (commercial silica gel spheres: $20-45 \mu \mathrm{m}$ and $3 \mu \mathrm{m}$, HMS spheres: $\sim 1.5 \mu \mathrm{m}$ ) were used as core templates. A polycrystalline silicalite-1 shell was formed upon first covering the external surface of the core templates with discrete silicalite-1 nanocrystals via electrostatic attractions, followed by short hydrothermal treatments in silica/TPAOH-containing gel to ensure shell coverage and uniformity. The synthesized materials were characterized SEM, TEM, XRD and nitrogen physisorption. Series of batch glycerol adsorption experiments were conducted to evaluate the ability of the final product in the selective removal of free glycerol from crude biodiesels with different compositions at various temperatures. Glycerol contents of the produced biodiesel were compared to those purified by using conventional adsorbents including bare mesoporous silica gel spheres, conventional zeolites, e.g. silicalite-1, pure siliceous beta (Si-BEA) and ZSM-5 $(\mathrm{H})$ crystals as well as physical mixture of the constitutive materials, i.e., equally mixed silicalite-1 and silica gel spheres. Although mesoporous silica gel spheres showed slightly higher glycerol adsorption capacity, the mesoporous adsorbents tend to trap a significant amount of bulkier molecules (e.g., FAME) in their large pore network $\left(d_{\text {pore }}=7 \mathrm{~nm}\right)$. However, the silicalite- 1 shell provided a microporous membrane which hindered FAME diffusion into the mesopores of the composite adsorbent, while the large pore volume of the mesoporous core enabled a multi-layer glycerol adsorption. This property of the core@shell material significantly enhanced the dry washing performance in terms of purification yield and adsorption capacity, in comparison to other conventional sorbents (Glycerol:FAME ratio in sorbent improved from $\sim 0.65$ for silica gel up to 5.2 for core@shell particles).
\end{abstract}

[a] Prof. F. Kleitz, N. Masoumifard Department of Chemistry, Université Laval, Quebec, G1V 0A6, QC Canada

E-mail: freddy.kleitz@chm.ulaval.ca

[b] Prof. S. Kaliaguine, N. Masoumifard

Department of Chemical Engineering, Université Laval, Quebec, G1V 0A6, QC, Canada

E-mail: serge.kaliaguine@gch.ulaval.ca

[c] Dr. P.M. Arnal

Centro de Tecnología de Recursos Minerales y Cerámica (CETMIC) CIC - CONICET La Plata

Centenario y 506, B1897ZCA, M. B. Gonnet, Argentina

[d] Prof. F. Kleitz, N. Masoumifard

Centre de Recherche sur les Matériaux Avancés (CERMA)

Université Laval, Quebec, G1V 0A6, QC, Canada

Supporting information for this article is given via a link at the end of the document.

\section{Introduction}

Composite materials in a core@shell structure with inherent properties, e.g., hierarchical porosity and diverse integrated functionalities, have recently attracted extensive research attention in many areas including adsorption and catalysis. ${ }^{[1]} \mathrm{A}$ typical core@shell consists of two different materials in such a way that one, the shell, entirely encompasses the inner compartment, the core (Scheme 1). Among all types of possible building materials for such composites, crystalline zeolites seem ideal for forming the shell of a core@shell sphere owing to their high thermal/hydrothermal stability, excellent resistance under corrosive conditions, highly ordered pore structure, large specific surface area and micropore volume, shape-selectivity and intrinsic chemical activity. Over the past fifteen years, a variety of core materials, such as polymers ${ }^{[2]}$, amorphous silica ${ }^{[3]}$, metal oxides ${ }^{[4]}$ and even different types of zeolites ${ }^{[4 a, 5]}$ were used to synthesize either core@shell or hollow materials with a zeolitic shell. These materials are mostly synthesized by applying a well-known strategy, the so-called layer-by-layer technique ${ }^{[6]}$, to cover the pre-synthesized solid core templates with desirable nanocrystals, which later grow through a hydrothermal treatment in a gel containing essential nutrients to strengthen the zeolitic shell and ensure uniform coverage. ${ }^{[2 a]}$

Application of zeolitic core@shell materials began with the pioneering work of Bouizi et al. ${ }^{[5 d]}$ who illustrated the superior performance of beta zeolite@silicalite-1 material in selective adsorption from a hydrocarbon mixture, containing butane, toluene and 1,3,5-trimethylbezene. In contrast to the smaller hydrocarbons, the bulkier 1,3,5-trimethylbenze molecules are inhibited from reaching to large pores of the $\beta$ zeolite core since the silicalite-1 shell presents a smaller pore size than their kinetic diameters. Since then, the core@shell materials with a zeolitic shell were used in different applications, primarily in the field of catalysis. ${ }^{[7]}$ The zeolitic shell provides an effective protective layer under harsh operating conditions of the reactions as well as a selective barrier against impurities, poisons and undesirable reactions, enhancing the catalyst activity, selectivity and durability. The beneficial aspects of utilizing zeolitic core@shell materials in gas phase adsorptive separation processes are also emphasized in a number of publications. ${ }^{[8]}$ However, the performance of the zeolitic core@shell materials still needs to be explored in other important liquid phase separation processes such as biodiesel purification, which involves considerable size difference between impurities and product molecules. Such a process is crucial for further development of sustainable chemicals and fuels.

Biodiesel, which usually refers to fatty acid methyl esters (FAME), is a renewable and environmentally friendly source of fuel for diesel engines. It is frequently produced at industrial scale by alkali-catalyzed transesterification of triglycerides from 
vegetable oil and animal fats with methanol. After the reaction, crude biodiesel is separated from the polar by-product, mainly glycerol, by either centrifugation or gravity settling process. Crude biodiesel can be neutralized at this step by adding a mineral acid to eliminate the soap from crude biodiesel. The methanol remaining in crude biodiesel which increases the solubility of glycerol, may be removed by vacuum/flash evaporation. After all these steps, crude biodiesel still contains different types of contaminants including unconverted triglycerides (TG), diglycerides (DG), monoglycerides (MG), free fatty acids (FFA), glycerol, water, catalyst, soaps, salts and others which should be totally or partially removed. Among them the removal of the free glycerol is of a great importance for its negative effects on biodiesel storage and usage such as settling problems, fuel tank bottom deposits, injector fouling, engine durability issue and higher emission of aldehydes and acrolein. Maximum allowable concentration of free glycerol, established by ASTM D6751 and EN 14214, is 0.02 wt\% in a pure finished product. ${ }^{[9]}$ Traditionally, glycerol is removed through extraction with water. Despite many advantages of the water washing process, it creates a number of problems mostly due to the need of large amounts of deionized water, wastewater management, biodiesel drying, significant product loss, time-consuming process, emulsion formation and corrosion. ${ }^{[10]}$

There is an alternative water-free adsorption-based process for biodiesel purification, known as dry washing, which is more ecofriendly and cost-effective than traditional water washing process. Dry washing eliminates contaminants by keeping the crude biodiesel in contact with an adsorbent or an ion-exchange resin. Numerous different adsorbent materials were previously studied for treating crude biodiesel. Relevant considerations, similarities, pros and cons found for all existing adsorbents are discussed in details somewhere else. ${ }^{[10,11]}$ Among available adsorbents, the hydrophilic ones such as silica and magnesium silicate are most actively investigated for the refining of biodiesel due to the fact that the biodiesel impurities are mostly polar compounds. Having high saturation capacity for glycerol and other polar impurities, silica gel particles are predominantly used for biodiesel purification either in a batch adsorber ${ }^{[12]}$ or in a fixed bed ${ }^{[13]}$. Although faster diffusion of the glycerol within the large pores of silica gel (pore sizes larger than $7 \mathrm{~nm}$ ) can potentially achieve significant glycerol removal in an adsorptive separation process, it is shown that using such an adsorbent leads to a perceptible reduction in purification yields. ${ }^{[14]} \mathrm{A}$ part of the esters is lost during the course of purification with almost all traditional adsorbents due to the capturing FAME along with unwanted impurities, decreasing the final yield. ${ }^{[15]}$ This reduction becomes more significant with applying larger doses of adsorbent.

One solution can be using adsorbents with smaller pores, e.g. microporous zeolites. The separation power of a zeolite strongly depends on micropore characteristics. In general, high selectivity is achieved when the pore size is comparable to the kinetic diameters of the molecules to be separated. Despite this appealing feature of zeolites, several important issues continue to limit the application of such materials as adsorbents in biodiesel purification systems. The smaller pore size is often coupled with lower adsorption capacity due to the relatively low pore volume. Moreover, intracrystalline transport limitation is another disadvantage, which imposes serious problems to process productivity, even in large pore zeolites. ${ }^{[8 c]}$

With these considerations in mind, it was hypothesized that a novel class of composite material, namely core@shell architecture, consisting of high surface area, high pore volume mesoporous silica spheres covered with a shape-selective zeolite, may hold considerable promise in biodiesel purification applications with regard to selective glycerol removal in high purification yield. Therefore, the present contribution reports the synthesis of mesoporous silica@zeolite via deposition of silicalite-1 nanocrystals over mesoporous silica spheres with various particle and pore sizes followed by a secondary hydrothermal treatment step. The performance of the synthesized sorbents was evaluated at different operating conditions by conducting free glycerol removal tests from crude biodiesel.

\section{Results and Discussion}

\section{Synthesis and Characterization of the Sorbents}

Microporous/mesoporous core@shell materials were synthesized by using a method similar to that described by Bouizi et al. for $\beta$-zeolite@silicalite-1 composites. ${ }^{[5 \mathrm{~d}]}$ Commercial silica gel spheres with two different sizes and HMS microspheres were used as core materials, which were ultimately covered with a polycrystalline intergrown silicalite-1 shell (Scheme 1). The silicalite-1 shell could not be placed by a simple one-pot hydrothermal crystallization which is used to produce bulk crystals. A successful coverage was only possible through a multistep synthesis route involving preliminary adsorption of zeolite nanocrystals onto large core particles followed by growing these nanocrystals in an appropriate synthesis gel mixture. Chemical and hydrothermal stability of the core as well as rapid growth of the nanocrystals were vital parameters toward the formation of the core@shell material. ${ }^{[7,16]}$

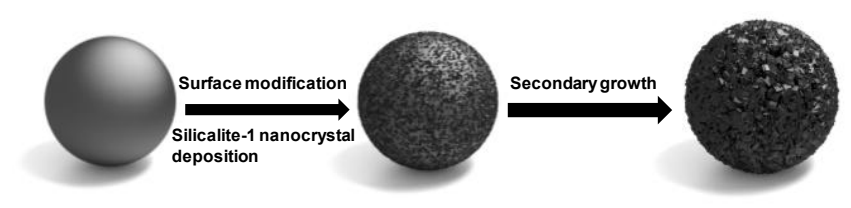

Scheme 1. Schematic representation of the mesoporous silica@silicalite1 synthesis

Particle size distribution and morphology of the silicallite- 1 and mesoporous silica spheres were first studied using transmission electron microscopy (TEM) and scanning electron microscopy (SEM) (Figure 1). Discrete silicalite-1 nanocrystals (Figure 1a) showed a narrow crystal size distribution with a mean diameter of $70 \mathrm{~nm}$ and a polydispersibility index of 0.025 as measured by Zetasizer particle analysis (Figure S1, Supporting information). The synthesized HMS particles were quite uniform in size and 
shape with mean diameter around $1.5 \mu \mathrm{m}$ as confirmed by SEM observation (Figure 1b). Two commercial silica gel spheres SG3 and SG20 (Silicycle Inc, Canada), showed wider particle size distributions than HMS, especially SG20 with a size range of 20 to $45 \mu \mathrm{m}$ (Figures 1c and 1d). All mesoporous silica spheres provided a smooth external surface area as seen in Figure 1, which makes them ideal core template for coating purposes.

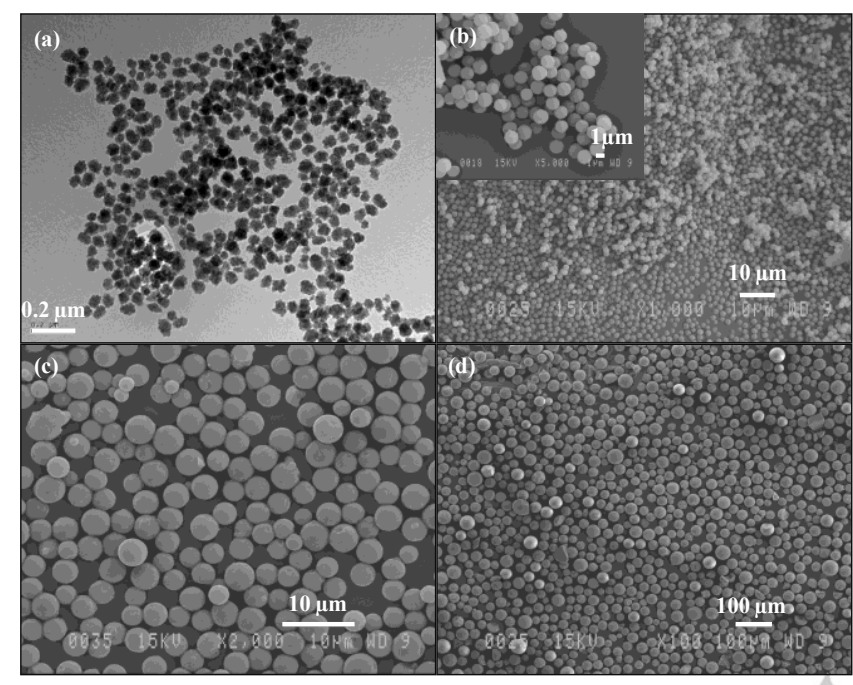

Figure 1. (a) TEM image of silicalite-1 nanocrystals (Scale bar $=0.2 \mu \mathrm{m}$ ), and SEM images of (b) HMS microspheres (Scale bar=10 $\mu \mathrm{m}$ ), (c) silica gel spheres $3 \mu \mathrm{m}$ (SG3) (Scale bar=10 $\mu \mathrm{m}$ ) and (d) silica gel spheres 20-45 $\mu \mathrm{m}$ (SG20) (Scale bar=100 $\mu \mathrm{m}$ ). Inset shows a higher magnification image.

Figure 2 shows two different core particles after coating by a uniform and closed-packed layer of silicalite-1 nanocrystals. Following two successive hydrothermal treatments of 45 min each in TEOS/TPAOH containing gel, the shell, which is initially formed by deposited zeolite nanocrystals, became a continuous, well-intergrown compact layer without any visible cracks or defects on the outer surface.

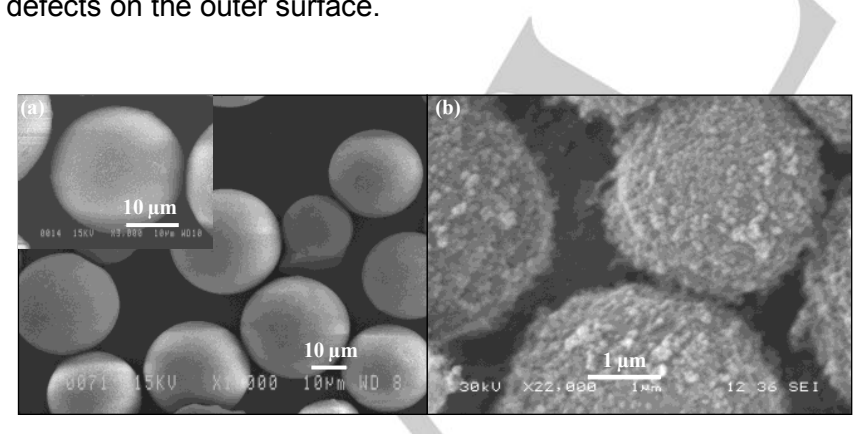

Figure 2. SEM image of covered (a) SG20 spheres (Scale bar=10 $\mu \mathrm{m}$ ) and (b) SG3 spheres with silicalite-1 nanocrystals using layer-by layer technique (Scale bar= $1 \mu \mathrm{m})$. Inset shows corresponding higher magnification image.

$$
4
$$

Figure 3 shows SEM images of the core@shell particles with different core sizes. The top surface of a core@shell particle can be clearly seen from the higher magnified inset images.
Subtracting the average size of core@shell particles from the average size of the core particles, the average shell thickness were estimated, reported in Table 1 , with the exception of SG20@silicalite-1 due to the non-uniform particle size distribution of parent particles (SG20).

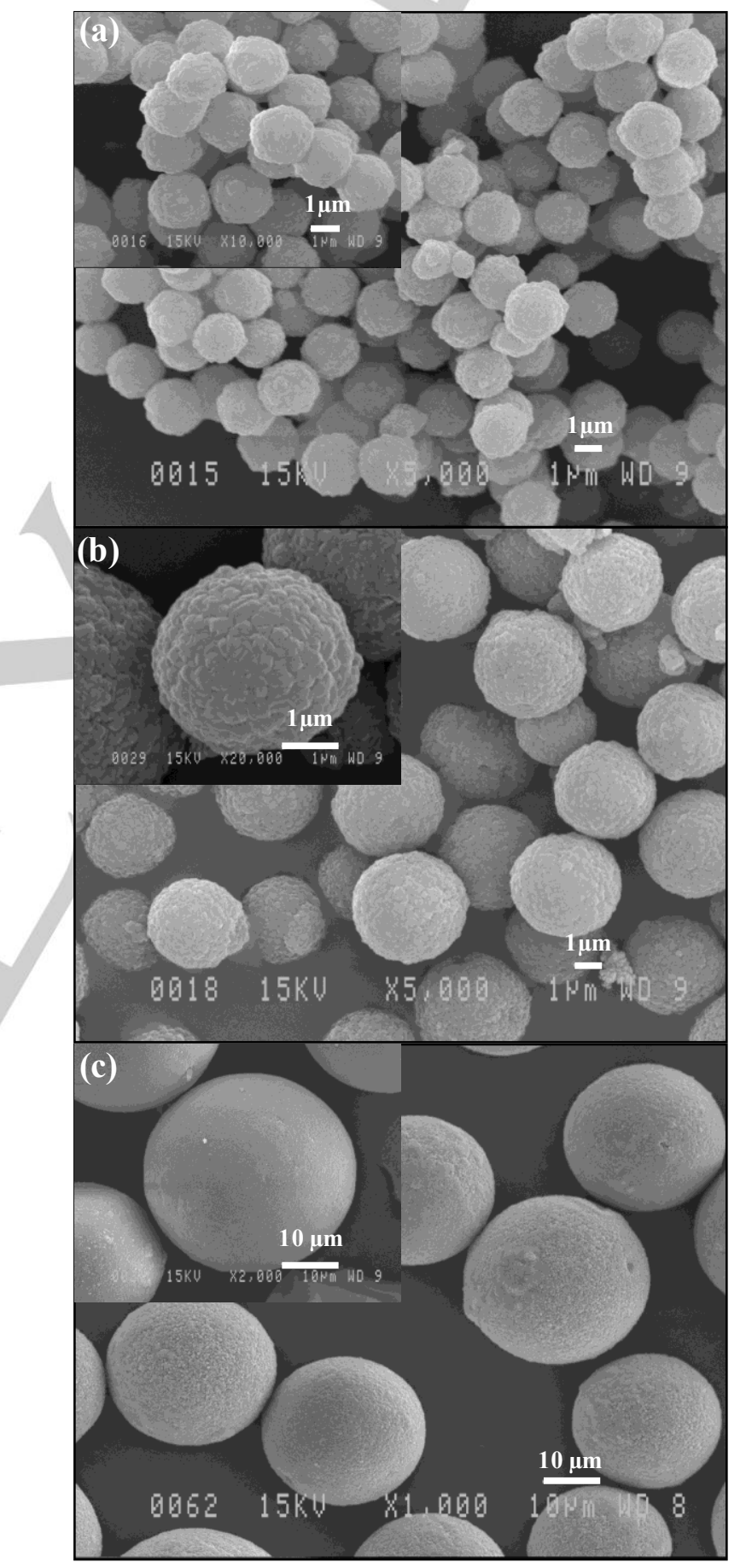

Figure 3. SEM image of (a) HMS@silicalite-1 (Scale bar=1 $\mu \mathrm{m}$ ), (b) SG3@silicalite-1 (Scale bar=1 $\mu \mathrm{m}$ ) and (c) SG20@silicalite-1 (Scale bar= 10 $\mu \mathrm{m})$. Inset shows corresponding higher magnification image. 
Table 1. Textural properties of all the adsorbent materials, obtained by performing $\mathrm{N}_{2}$ physisorption analysis at $-196^{\circ} \mathrm{C}$

\begin{tabular}{|c|c|c|c|c|c|c|c|c|}
\hline Sample & $\begin{array}{l}\mathrm{S}_{\mathrm{BET}}{ }^{[\mathrm{a}]} \\
\mathrm{m}^{2} / \mathrm{g}\end{array}$ & $\begin{array}{l}\text { Micropore } \\
\text { surface } \\
\text { area }^{[b]} \\
\mathrm{m}^{2} / \mathrm{g}\end{array}$ & $\begin{array}{l}\text { Micropore } \\
\text { volume }{ }^{[\mathrm{c}]} \\
\mathrm{cm}^{3} / \mathrm{g}\end{array}$ & $\begin{array}{l}\text { Pore } \\
\text { volume } \\
\mathrm{cm}^{3} / \mathrm{g}\end{array}$ & $\begin{array}{l}\text { Maxima } \\
\text { mesopore } \\
\text { size } e^{[\mathrm{e}]} \\
\mathrm{nm}\end{array}$ & $\begin{array}{l}\text { Micropore } \\
\text { size }^{[f]} \\
\mathrm{nm}\end{array}$ & $\begin{array}{l}\text { Shell } \\
\text { thickness }^{[g]} \\
\mu \mathrm{m}\end{array}$ & $\begin{array}{l}\text { Shell } \\
\text { coverage }\end{array}$ \\
\hline HMS & 780 & ---- & ---- & 0.63 & 3.3 & --- & & ---- \\
\hline Silica gel $(3 \mu \mathrm{m})$ - SG3 & 453 & ---- & --- & 0.7 & 6.2 & ---- & ---- & --- \\
\hline Silica gel $(20-45 \mu \mathrm{m})-\mathrm{SG} 20$ & 326 & --- & --- & 0.79 & 6.4 & ---- & ---- & ---- \\
\hline HMS@silicalite-1- Non-calcined & 8.9 & --- & 0 & 0.017 & --- & ---- & --- & ---- \\
\hline HMS@silicalite-1- Calcined & 430 & 335 & 0.13 & 0.26 & $3.7,6$ & $\begin{array}{l}0.51 \times 0.55 \\
0.53 \times 0.56\end{array}$ & 0.4 & 99 \\
\hline SG3@silicalite-1- Non-calcined & 11.5 & --- & 0 & 0.027 & ---- & ---- & ---- & ---- \\
\hline SG3@silicalite-1- calcined & 280 & 166 & 0.08 & 0.25 & $3.5,7.2$ & $\begin{array}{l}0.51 \times 0.55 \\
0.53 \times 0.56\end{array}$ & 0.7 & 97 \\
\hline SG20@silicalite-1- Non-calcined & 3 & --- & 0 & 0.006 & -- & --- & --- & --- \\
\hline SG20@silicalite-1- calcined & 180 & 70 & 0.025 & 0.24 & $3.5,7.4$ & $\begin{array}{l}0.51 \times 0.55 \\
0.53 \times 0.56\end{array}$ & ---- & 99 \\
\hline Si-beta zeolite & ---- & 608 & 0.24 & ---- & ---- & $\begin{array}{l}0.66 \times 0.67 \\
0.56 \times 0.56\end{array}$ & ---- & ---- \\
\hline Al-ZSM-5 (H) & ---- & 300 & 0.14 & ---- & --- & $\begin{array}{l}0.51 \times 0.55 \\
0.53 \times 0.56\end{array}$ & --- & --- \\
\hline Silicalite-1 & 484 & 378 & 0.15 & 0.55 & 16 & $\begin{array}{l}0.51 \times 0.55 \\
0.53 \times 0.56\end{array}$ & --- & --- \\
\hline
\end{tabular}

[a] Calculated by using the BET method on relatively low-pressure region $\left(P / P_{0}=0.05-0.2\right)$. [b] Difference between $S_{B E T}$ and NLDFT cumulative surface area for pore sizes larger than $2 \mathrm{~nm}$. [c] Calculated using NLDFT cumulative pore volume for pore sizes smaller than $2 \mathrm{~nm}$. [d] Calculated at $P / \mathrm{P}_{0}=0.95$. [e] Derived from NLDFT pore size distribution in mesopore region (pore sizes larger than $2 \mathrm{~nm}$ ). The two values correspond to the two maxima given by the pore size distribution curves. [f] Micropore sizes for pure zeolites and zeolitic part of the core@shell particles were derived from IZA website. [g] Estimated from SEM images.

Figure $4 \mathrm{a}$ shows the isotherms, obtained by nitrogen sorption measurements for the various materials: (i) mesoporous silica

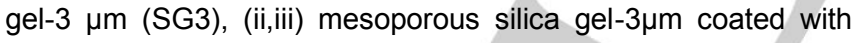
silicalite-1 nanocrystals prior to and following the final calcination, and (iv) pure silicalite- 1 submicron crystals. A large $N_{2}$ uptake and a hysteresis loop at high relative pressures $\left(P / P_{0}>0.8\right)$ was observed for silicalite-1 sub-micron crystals, which is related to the adsorbate condensation in large mesopores (pore sizes $\sim 16$ $\mathrm{nm}$ ) formed upon aggregation of small crystals during drying and calcination. The silica core showed a type IV nitrogen adsorption-desorption isotherm, characteristic of mesoporous solids, with an uptake in the relative pressure region of $0.6-0.8$. Two successive secondary growth of the silicalite-1 nanocrystal layer, deposited on the external surface of the mesoporous silica spheres, led to a uniform shell of $\mathrm{TPA}^{+}$-containing silicalite- 1 . This stuffed zeolitic layer efficiently restricts the access of nitrogen molecules to the channels of the mesoporous silica core, as confirmed by a low $\mathrm{N}_{2}$ uptake of the non-calcined core@shell material. The same behaviors were also observed for the bare HMS and SG20 core particles and the uncalcined respective core@shell materials (Figures S6 and S4, Supporting information)

Wang et al. ${ }^{[7]}$ showed that the reduction in $\mathrm{N}_{2}$ uptake cannot be due to either the pore filling of the core, $\beta$ zeolite in their case, by organic TPAOH molecules used in secondary growth gel nor the formation of silicalite- 1 crystals as a separate phase in a mixture with the core. They obtained comparable surface area between an untreated calcined $\beta$ zeolite and a treated one under similar conditions as the secondary growth step, i.e., exposing to TPAOH-containing clear gel for a certain period of time, washing and drying. Considering this result and the fact that mesoporous silica gel (used as the core in the present study) exhibit much larger pore sizes (around $7 \mathrm{~nm}$ ) than $\beta$ zeolite (around $0.65 \mathrm{~nm}$ ), the filling of the core by organic TPAOH template molecules during secondary growth can be disregarded as a plausible cause for the observed reduction in $\mathrm{N}_{2}$ uptake after the secondary growth step. Moreover, SEM imaging confirmed the absence of abundant silicalite-1 crystals in the bulk (Figure 3). All these results provide proof that the filled micropores of the shell have efficiently obstructed the path of $\mathrm{N}_{2}$ gas toward 
accessing the internal porosity of non-calcined core@shell products. This phenomenon has also been used by other researchers as a way to probe the integrity of the silicalite-1 shell around a zeolitic core by calculating the surface area ratio between the cores and the non-calcined core@shell samples. ${ }^{[5 d, 5 e, 7 e, 8 c]}$ The obtained values of BET surface area for non-calcined sample correspond to the rough external surface of the core@shell material since the internal pore network is not accessible. Using the same method revealed $97 \%$ to $99 \%$ of the mesoporous silica cores are coated with a uniform silicalite-1 layer after two successive secondary growth steps (Table 1).

(a)

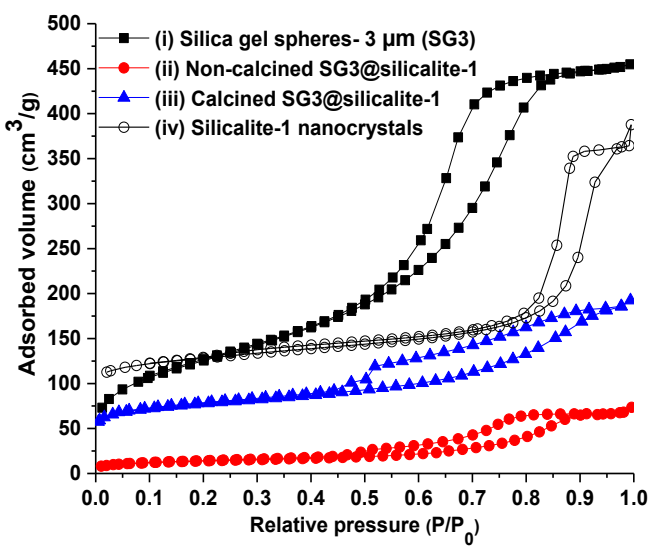

(b)

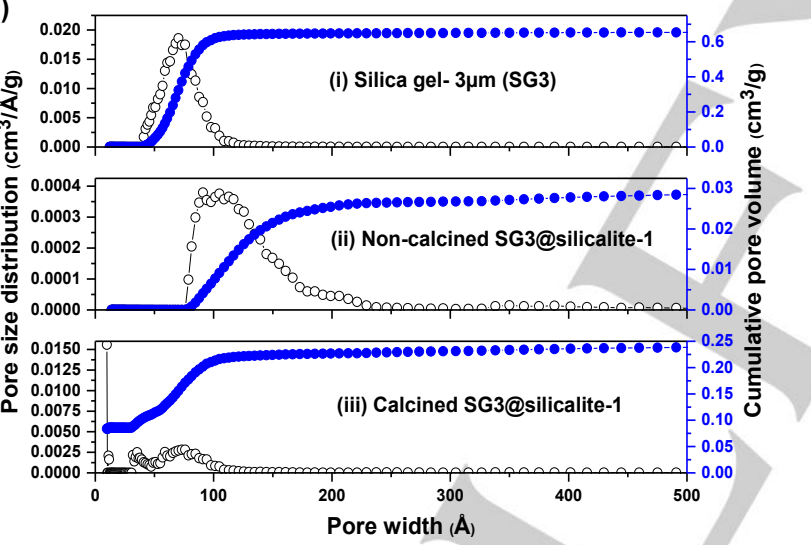

Figure 4. (a) Nitrogen adsorption-desorption isotherms measured at $-196{ }^{\circ} \mathrm{C}$

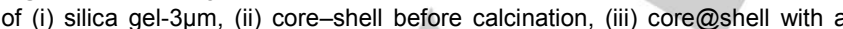
calcined shell, and (iv) silicalite-1 submicron crystals; (b) The corresponding NLDFT pore size distributions and cumulative pore volumes of (i) Silica gel-3 $\mu \mathrm{m}$ (SG3), (ii) non-calcined SG3@silicalite-1 (iii) calcined SG3@silicalite-1, calculated from the adsorption branch of the isotherm by using the NLDFT method.

The presence of both micropores and mesopores along with the specific connectivity in pore networks may account for the intricate $\mathrm{N}_{2}$ sorption behaviour of the calcined sample (Figure $4 \mathrm{a}$ Figures S6 and S4- Supporting information). The isotherm of the core@shell material showed a noticeable reduction in nitrogen uptake at higher values of $\mathrm{P} / \mathrm{P}_{0}$ (capillary condensation region), compared to the mesoporous silica spheres, since the mesopore contribution in the final porosity was drastically decreased by the dense microporous silicalite-1 shell. However, the capillary condensation can still be observed in the relative pressure region of 0.6-0.8 which is accompanied by a complex hysteresis. This capillary condensation indicates the presence of mesopores, originating from the well-defined core mesoporosity and the possible void spaces between the core and shell compartments. The hysteresis loop was built with a two-step desorption isotherm which shows the occurrence of normal equilibrium evaporation as well as a cavitation effect, obvious from a step down at relative pressures between 0.4-0.5 associated with hysteresis loop closure. ${ }^{[17]}$

The cumulative pore volumes and pore size distributions at different steps of the SG3@silicalite-1 synthesis are obtained by using NLDFT method $\left(\mathrm{N}_{2}\right.$ sorption in cylindrical silica pores, adsorption branch) and depicted in Figure $4 \mathrm{~b}$. The mesoporous silica gel spheres presented the largest pore volume and a narrow pore size distribution with a maximum centered at $7 \mathrm{~nm}$. For non-calcined core@shell materials, the pore volume has drastically decreased because the mesopores were blocked by $\mathrm{TPA}^{+}$-filled microporous silicalite- 1 shell. However, negligible void spaces were observed which can be due to the presence of either few uncoated silica particles or the intercrystalline spaces, formed between intergrown silicalite-1 within the polycrystalline shell. Upon calcination, two different types of pores appeared in the pore size distribution contributing to the final pore volume; pores smaller than $2 \mathrm{~nm}$ corresponding to the microporous crystalline shell and pores larger than $2 \mathrm{~nm}$ which were primarily associated to the mesopore core compartment. Considering the limitation of nitrogen adsorption at $-196{ }^{\circ} \mathrm{C}$ on quantitative evaluation of microporosity, especially in the range of ultramicropores of silicalite-1 (pore widths $\sim 0.55 \mathrm{~nm}$ ), more advanced analyses using argon as an adsorptive at $-186^{\circ} \mathrm{C}$ will be useful for further investigation of this system.${ }^{[17 a]}$ Regarding the second types of pores, i.e., mesopores, a wide pore size distribution in the mesopore region was observed, showing two main peak maxima centered around $4 \mathrm{~nm}$ and $7 \mathrm{~nm}$, respectively. The second peak around $7 \mathrm{~nm}$ originates from the silica gel particles as one of the main building blocks. The first peak, however, can be an artifact associated with the complex pore network connectivity between the mesoporous core and microporous shell. In general, the $\mathrm{N}_{2}$ sorption results did not fully comply with the observed mesoporosity of the parent silica particles owing to the structural changes of the core during either secondary growth or high temperature calcination steps. Similar trends for pore size distributions of both HMS@silicalite1 and SG20@silicalite-1 were observed, as illustrated in Figures S5 and S7. Textural properties of the different calcined and noncalcined core@shell spheres along with the calcined mesoporous silica cores obtained by nitrogen physisorption measurement are summarized in Table 1 . The average pore sizes of the adsorbents were derived from either $N_{2}$ sorption measurements for mesoporous materials including core@shell and silica spheres or from tabulated data (IZA website) for microporous pure zeolitic or core@shell adsorbents. ${ }^{[18]}$

The XRD patterns obtained for building materials, i.e. silicalite-1 nanocrystals and HMS spheres, as well as core@shell products before and after secondary growth steps are shown in Figure 5. 
The same patterns were recorded for other core@shell materials with silica gel-type cores and reported in Figures S8 and S9. Weak characteristic peaks of the silicalite-1 zeolite were observed on the XRD profile of the silica cores after depositing silicalite-1 nanocrystals (Figure $5 b$ ) due to the small quantity and the size of the adsorbed nanocrystals. After hydrothermal treatment (Figure $5 \mathrm{c}$ ), however, the more intense peaks of the MFI phase at $2 \theta=7.5-9.58^{\circ}$ and $23-24^{\circ}$ emerged. Nevertheless, the preferred growth orientation cannot be identified from the obtained pattern due to initial random packing of the silicalite-1 crystals over the mesoporous silica surface. In addition, the peak of mesoporous silica was apparent at lower angles in core@shell product, which confirms the silica cores were all encapsulated by zeolites, and survived under the harsh environment of secondary growth condition. ${ }^{[16]}$ This was also confirmed by nitrogen sorption measurement of core@shell material. All these evidences endorse that the mesoporous silica@silicalite-1 structure were successfully synthesized.

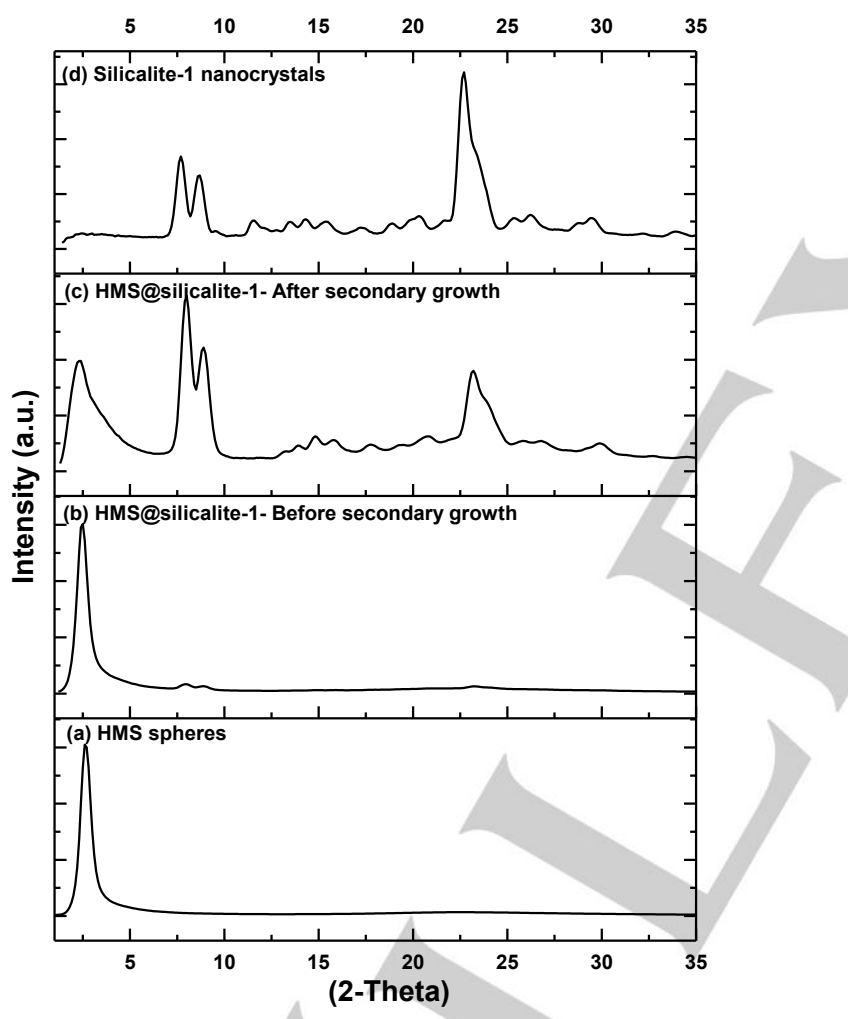

Figure 5. Wide-angle powder XRD patterns for (a) bare HMS, (b) coated HMS before hydrothermal treatment, (c) core-shell particles after secondary growth and (d) silicallite-1 nanocrystals.

\section{Purification of the Crude Biodiesel}

Table 1 summarizes the physical properties of all adsorbents used to remove free glycerol from crude biodiesel. These adsorbents can be categorized into three different classes of materials regarding their pore structures; (1) mesoporous silica with an average pore diameter of 3.5 for HMS spheres and $7 \mathrm{~nm}$ for commercially-available silica gel particles, which are significantly larger than the molecular dimension of FAME and glycerol. As none of these materials can provide shape selectivity for glycerol adsorption, the adsorption study with mesoporous adsorbents was performed using the commercially available silica gel spheres, (2) microporous zeolite crystals, i.e., Si-BEA and Al-ZSM-5 $(\mathrm{H})$ microcrystals, as shown in Figures S2 and S3, respectively. This category provides pore sizes comparable to the dimension of molecules present in biodiesel, (3) core@shell material with large mesopores in the core and small micropores in the surrounding shell. The corresponding micropore entrances over the shell surface are capable of sieving molecules and allowing a selective sorption from a mixture on the basis of their kinetic diameters. In order to evaluate the glycerol removal ability of adsorbents in presence of different contaminants, crude biodiesels with two different compositions were used; (1) a methanol-free biodiesel and (2) a methanol-containing biodiesel (methanol content $\sim 0.7 \mathrm{wt} \%$ ). The equilibrium content of hydrophilic glycerol in biodiesel is a function of different parameters, such as temperature and other impurities, including residual methanol and amphiphilic monoand diglycerides. ${ }^{[12 b]}$ Free glycerol contents of methanol-free and methanol-containing biodiesels were around $0.065 \mathrm{wt} \%$ and $0.22 \mathrm{wt} \%$, respectively. The effect of temperature as one of the key parameters on biodiesel dry washing was also studied by performing the adsorption tests at $25^{\circ} \mathrm{C}$ and $100{ }^{\circ} \mathrm{C} .{ }^{[19]}$

\section{Glycerol adsorption from methanol-free biodiesel}

Figure 6 compares the glycerol adsorption capacities at equilibrium for different adsorbents using methanol-free biodiesel. The key role of the presence of mesopores on glycerol adsorption is clear from the glycerol uptake at equilibrium for silica gel and core@shell spheres; the one with highest mesopore surface area, $3 \mu \mathrm{m}$ silica gel spheres, showing the maximum glycerol adsorption capacity. In addition, the smaller silica gel spheres could probably provide better contact with biodiesel constituents which facilitate their diffusion to reach adsorption sites within the particles. Conventional zeolites, SiBEA and Al-ZSM-5 (H) microcrystals, showed the lowest glycerol adsorption capacities with only minor differences, even though higher glycerol uptake by $\beta$ zeolite was expected due to its higher micropore volume than Al-ZSM-5 $(H)$. Surface chemistry provides a plausible explanation; it is known that the synthesis of zeolites via fluoride route, e.g., pure siliceous $\beta$ zeolite (Si-BEA) in this study, provides a well-defined crystalline structure with less framework defects ( $\mathrm{SiO}^{-}$or $\mathrm{SiOH}$ groups) on the surface. ${ }^{[20]}$ As a consequence, the Si-BEA crystals exhibit higher hydrophobicity compared to the zeolite synthesized in a basic medium. In addition to the presence of more silanol groups on the surface, the synthesized ZSM-5 zeolite (Al-ZSM-5 $(\mathrm{H})$ ) benefits from very strong acid-sites owing to the presence of $\mathrm{H}^{+}$, neutralizing the surface negative charges caused by intraframework aluminum atoms. This situation provides better 
conditions for the adsorption of polar molecules such as glycerol on the surface through dipole-field interaction and hydrogen bonding. However, in contrast to mesoporous silica, the zeolite microcrystals in general suffer from small micropore volume, small external surface area and tiny pore mouth, all contributing to poor performance of conventional zeolites for this application. Another interesting feature of mesoporous adsorbents such as silica gel particles is related to the large internal pore spaces which permit a multi-layer glycerol adsorption via hydrogen bonding on their silanol-rich surfaces.

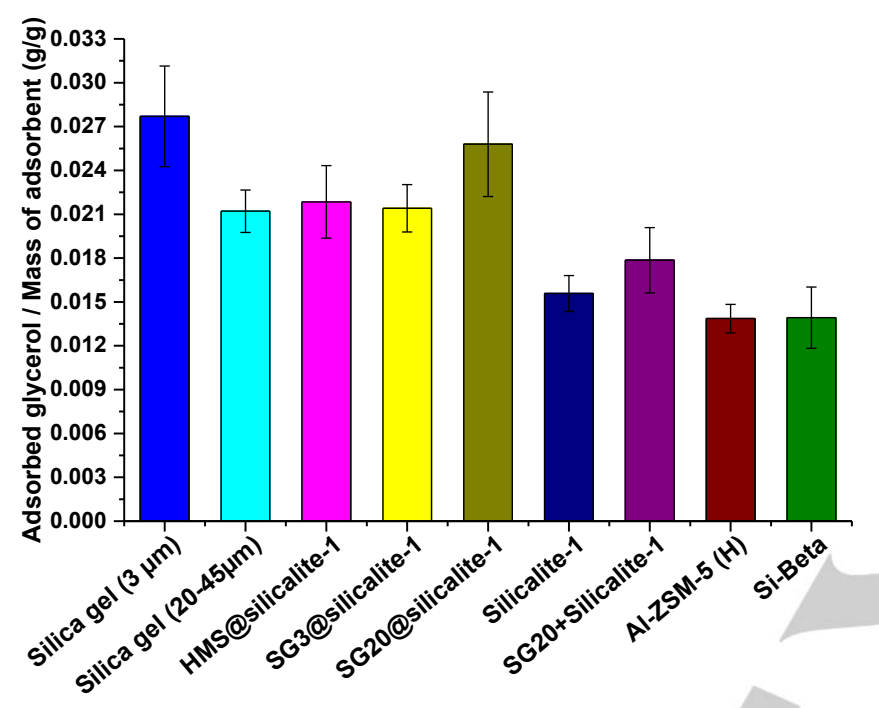

Figure 6. Specific adsorption loading of glycerol at equilibrium for different adsorbents using methanol-free biodiesel at $25 \mathrm{C}$ (glycerol initial concentration in biodiesel $=0.065 \mathrm{wt} \%$, adsorbent concentration= $2.3 \mathrm{wt} \%$ ).

Covering $3 \mu \mathrm{m}$ silica gel spheres with silicalite-1 caused a significant decrease in glycerol adsorption capacity. This could be a direct consequence of reduced mesoporosity of the core@shell materials. Therefore, the adsorption capacity may be partially restored by increasing the mesopore contribution to the total porosity of a core@shell material. One strategy is to use larger core particles. By doing so, SG20@silicalite-1 was tested and it showed higher glycerol adsorption than other core@shell materials, being even higher than that of large silica gel spheres. This can be attributed to the greater mesopore volume which is the highest among all synthesized core@shell materials. Since the accessibility to mesopores in core@shell structures is effectively restricted by the silicalite-1 shell, small molecules such as glycerol will diffuse faster than bulkier molecules (FAME soap, MG, DG, TG) and fill the pores by multilayer adsorption. It is worth mentioning that although the presence of overlapping error bars (with a 95\% level of confidence), especially for SG20 and all core@shell samples, make it difficult to presume the observed trend as statistically significant, the conclusion, i.e., larger cores improve glycerol adsorption of core@shell materials remains valid. Employing an equal-mixture of $20 \mu \mathrm{m}$ silica gel spheres and silicalite- 1 sub-micron crystals (around $300 \mathrm{~nm}$ ) as adsorbent resulted in no improvement in glycerol adsorption. Lower glycerol adsorption of this mixture compared to the core@shell adsorbents also proved that silicalite- 1 crystals as a shell play a positive role as shape-selective barrier and improves glycerol adsorption.

Total organic loadings, i.e., accumulated organic molecules within the pores of each adsorbent upon the termination of the purification process, were measured by running a TGA-DSC experiment over a temperature range of $35-700^{\circ} \mathrm{C}$. The TGA profiles are shown in Figure 7 . At temperature above $150{ }^{\circ} \mathrm{C}$, a two-step mass loss was observed for most of the samples, the first temperature-dependent mass loss with a slight slope occurred at temperature below $300{ }^{\circ} \mathrm{C}$, attributed to the vaporization of FAME from the outside of the pores (interparticle spaces/ external surfaces of the particles). The quite significant second mass-loss at temperature higher than $300^{\circ} \mathrm{C}$, can be assigned to a simultaneous volatilization/decomposition of high boiling point molecules including FAME, TG, DG, MG, FFA and free glycerol. ${ }^{[21]}$ Even though selective adsorption of polar components should be dominant on silica surfaces, it is evidenced by Figure 7 that a large number of non-polar/less polar materials enters inside the large void spaces provided by the mesopores of the bare mesoporous silica particles. On the other hand, a complete displacement of the air from within the pores with bulkier molecules, i.e., pore intrusion, is happening along with the adsorption of trace amount of glycerol. FAME and FFA molecules can be adsorbed via hydrogen bonding to the surface oxygen atoms mainly through carboxylate ion and ester carbonyl groups, respectively. ${ }^{[22]}$ This phenomenon limits the effectiveness of large pore adsorbents by decreasing the glycerol adsorption capacity and the biodiesel purification yield. Subtracting the mass of adsorbed glycerol obtained from GCFID from the total organic loading of adsorbent from TGA experiment, the uptake of bulkier organic molecules from the biodiesel mixture can be calculated. Note that, it is assumed that a quick washing with hexane right after the adsorption removes all biodiesel components from the bulk without eluting adsorbed molecules within the pores. The mass loss at temperatures higher than $150{ }^{\circ} \mathrm{C}$ is used for the calculation, since the freebiodiesel molecules start to evaporate at temperatures higher than $150^{\circ} \mathrm{C}$, depending on the oil used for biodiesel production.

Calculated FAME uptakes for different samples are presented in Figure 8 . It can be seen that the silica gel particles have the highest specific loading of bulkier molecules. The fact that the cross-sectional diameters of the FAME and glycerol molecules $(<0.5 \mathrm{~nm})$ are smaller than the average pore sizes of mesoporous silica spheres, listed in Table 1, justifies the observed behavior. This means that all molecules in crude biodiesel mixture, even TG, could diffuse through the extra-large pore size of the silica gel particles (around $7 \mathrm{~nm}$ ), and these adsorbents demonstrate no size exclusion effects, even if there is no tendency for non-polar molecules to be adsorbed on the polar silica surface. 


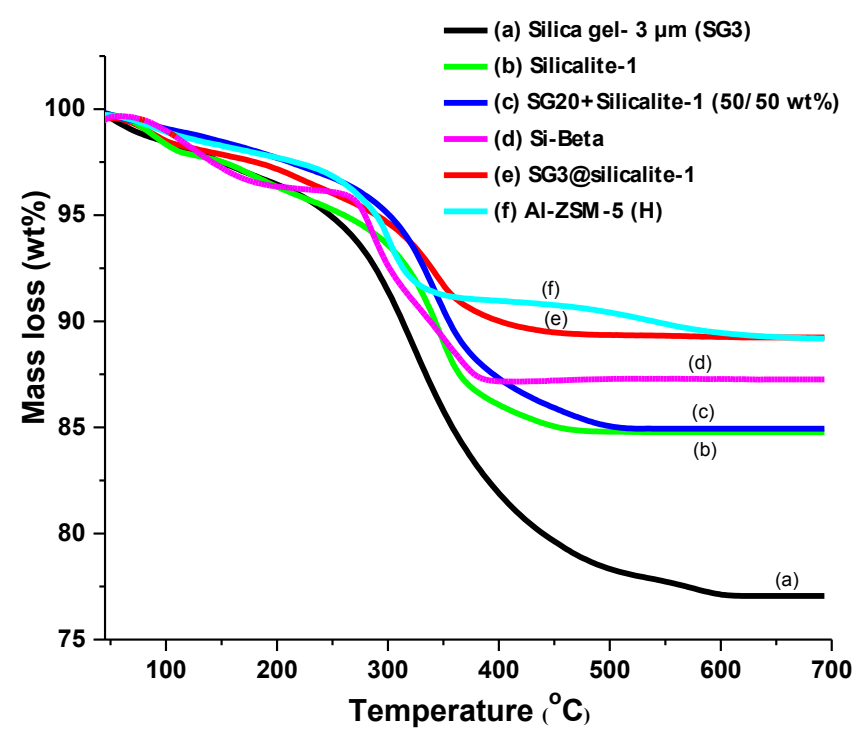

Figure 7. Overlay of thermogravimetric curves for the used adsorbents (glycerol initial concentration in biodiesel $=0.065 \mathrm{wt} \%$, adsorbent concentration $=2.3 \mathrm{wt} \%$ ).

Figure 8 also reveals that even conventional zeolites can accommodate large molecules such as FAME, possibly crawling inside. However, this would need longer time to reach equilibration. Although FAME molecules have a very long aliphatic hydrocarbon chain, they have a small cross-sectional molecular diameter. It is found that the effect of molecular diameter on hydrocarbon diffusion through a microporous zeolite is much more pronounced than that of the molecular length. ${ }^{[23]}$ The possibility of FAME diffusion through the micropores of zeolites is in line with a number of studies on various types of zeolites used as adsorbents in the field of fats and oils for chromatographic separation of fatty acids and FAME from oil, or even separation of mixtures of cis and trans "geometrical" isomers of mono- and polyunsaturated FAME. ${ }^{[22,24]}$ Looking for a zeolite, which offers better sieving effect between bulky biodiesel molecules and glycerol, zeolites with MFI and BEA structures were tested. As can be seen in Figure 8, large pore zeolites, such as $\beta$ zeolite, were not able to efficiently separate glycerol from esters of fatty acids, possibly due to the pore size of these zeolites $(6.5 \AA)$ being large enough to retain the relatively large ester molecules, whilst the MFI type zeolite (5.5 $\AA$ ), microcrystals Al-ZSM-5 $(\mathrm{H})$, was more suitable for the separation. The size of the crystals seems to have an important role as well. As can be seen in Figure 8 , sub-micron silicalite-1 particles (300 $\mathrm{nm}$ ), synthesized by secondary growth of free nanocrystals in the same gel used for core@shell, showed very high loading of FAME, even higher than the Si-BEA, which is due to the interstitial meso/macro spaces, created between aggregated small particles upon calcination. As confirmed by $\mathrm{N}_{2}$ adsorption measurements, very large mesopores with a maximum centered at $16 \mathrm{~nm}$ were observed for silicalite-1 submicron crystals (Table 1). In contrast to this undesirable effect, silicalite-1 size reduction leads to shorter micropores and higher external surface area, resulting in a slight improvement in glycerol adsorption as observed in Figure 7. The dual effect of crystal size can be exploited toward the designing of a more efficient glycerol adsorbent by simply avoiding meso/macro gaps between small particles, which is possible through a secondary growth of nanocrystals while they are tightly packed on a support.

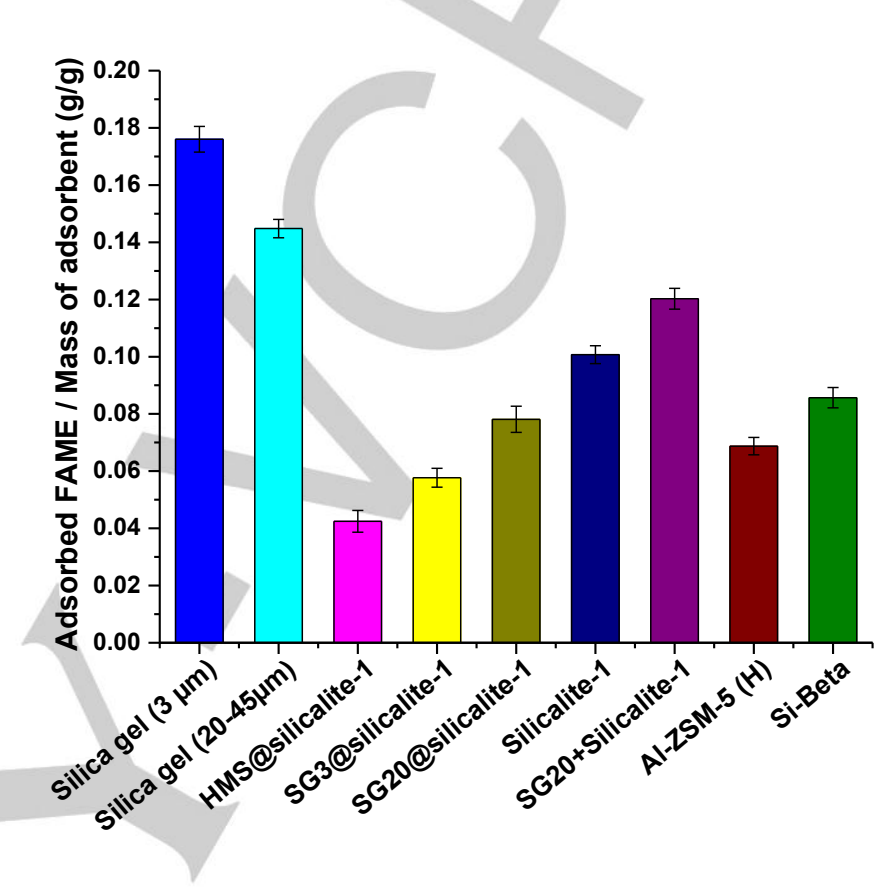

Figure 8. Specific uptake of FAME at equilibrium for different adsorbents using methanol-free biodiesel at $25{ }^{\circ} \mathrm{C}$ (glycerol initial concentration in biodiesel $=0.065 \mathrm{wt} \%$, adsorbent concentration= $2.3 \mathrm{wt} \%$ ).

In addition to the core mesoporosity, core@silicalite-1 benefits from shorter micropores (shell thickness $<1 \mu \mathrm{m}$ as estimated from SEM imaging) without providing any interstitial spaces, susceptible for adsorption of bulkier molecules. As can be seen in Figure 8, core@shell particles showed the lowest adsorption of bulkier molecules. Among the core@shell materials with different core sizes, HMS@silicalite-1 performed better regarding FAME adsorption, owing to the microporous coverage of this material. However, the contribution of core mesoporosity to the total porosity of HMS@silicalite-1 was the minimum compared to other core@shell materials (Table 1). It should be noted that a perceptible FAME uptake still occurs for all samples due to the long equilibrium time for the adsorption tests. However, the FAME uptake can be significantly reduced in the case of core@shell materials, considering the fact that the glycerol enters the pores of silicalite- 1 freely and from there to the core, but the bulkier molecules, like FAME, diffuse into the pores more slowly. In other words, finding an optimum contact time could significantly improve the efficiency of core@shell materials in real practical applications. 
Glycerol adsorption from methanol-free biodiesel at elevated temperature

Normally, a decrease in adsorption is expected by increasing temperature, however in the case of biodiesel, as a multicomponent mixture of FAME, FFA, TG, DG, MG, and glycerol, a temperature rise will have a complicated effect. Working at higher temperature decreases the viscosity of the mixture, thus the diffusion rates of both glycerol and bulkier molecules into the pore of adsorbents increases, especially for the microporous materials. Figure 9 suggests that upon heating to $100{ }^{\circ} \mathrm{C}$, FAME uptake in microporous silicalite-1 and the core@shell materials (equipped with a microporous shell) slightly increased, most likely because of better diffusion of bulkier molecules deep into the mesopores of the silica core. However, overlapping standard deviations indicate that the observed differences might not be statistically significant. The reverse behavior, a statistically significant decrease in FAME uptake, was observed for silica gel particles, complying with the theoretical expectation.

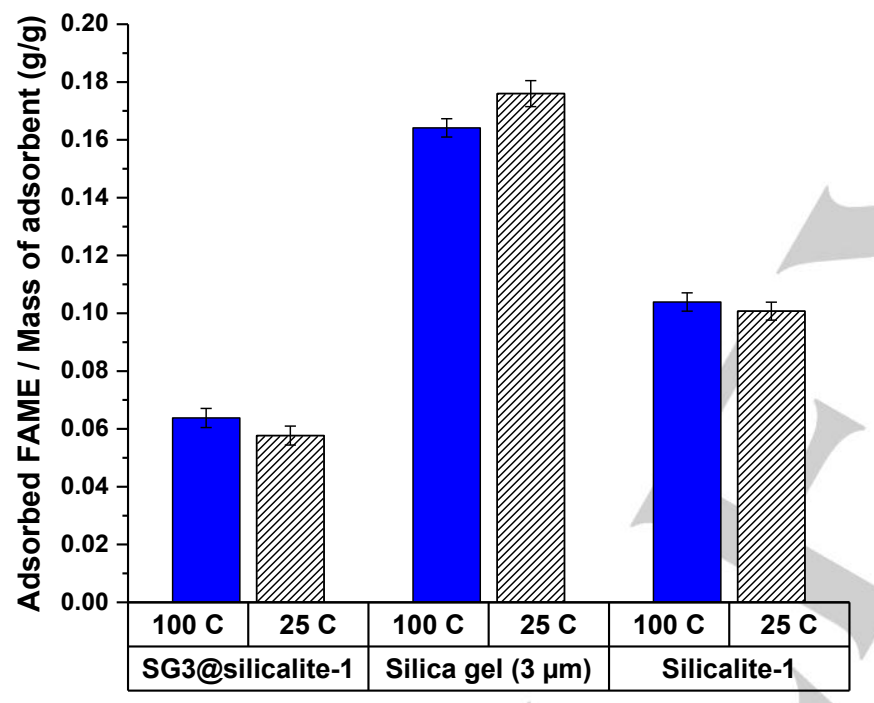

Figure 9. Uptake of FAME by different adsorbents from methanol-free biodiesel at different temperatures (glycerol initial concentration in biodiesel $=0.065 \mathrm{wt} \%$, adsorbent concentration= $2.3 \mathrm{wt} \%$ ).

Interestingly, as seen in Figure 10, glycerol adsorption at elevated temperatures followed the same trend as that of the FAME uptake. Apparently, a temperature increase facilitates the penetration of all the molecules, especially the smaller ones, and this, more significantly in microporous silicalite-1. This leads to desirable results for core@silicalite-1 materials by alleviating the diffusion limitations through micropores and thus allowing a multi-layer adsorption inside the core compartment.

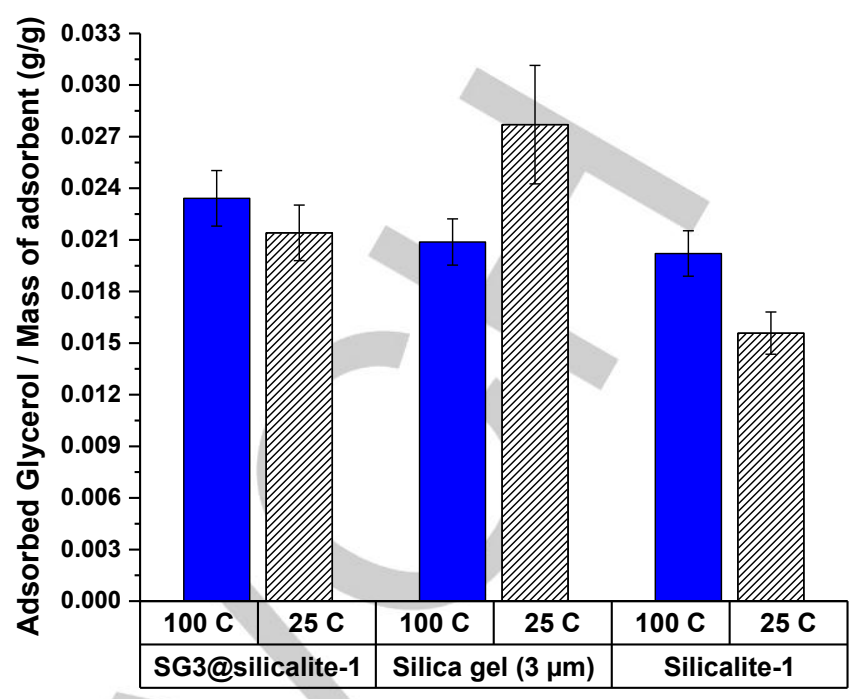

Figure 10. Adsorption of glycerol by different adsorbents from methanol-free biodiesel at different temperatures (glycerol initial concentration in biodiesel= $0.065 \mathrm{wt} \%$, adsorbent concentration= $2.3 \mathrm{wt} \%$ ).

Glycerol adsorption from methanol-containing biodiesel

According to literature, methanol negatively affects the adsorption of glycerol on silica surface because of an increased solubility of glycerol in FAME, as well as its higher affinity to the silica surface than glycerol. ${ }^{[10,12 b]}$ Although methanol has a negative effect on glycerol adsorption, it is economically and environmentally favorable to use adsorbents to purify biodiesel in its crude form right after glycerol separation by settling. Therefore, all adsorption tests were repeated for methanolcontaining biodiesel, which was produced by leaving biodiesel for $24 \mathrm{~h}$ at room temperature in a capped container after transesterification reaction to remove the main part of glycerol by-product by gravity separation. Average methanol contents of $0.7 \mathrm{wt} \%$ were measured for the methanol-containing biodiesel samples prior to adsorption test. Figure 11 shows the amounts of FAME and glycerol uptake from a methanol-containing biodiesel for different adsorbents. It was observed that the presence of methanol decreases the viscosity of biodiesel mixture. This presumably makes diffusion of the molecules easier; however, due to a large intrinsic interaction of inorganic adsorbents towards alcohols, the pore space was occupied by methanol and glycerol, present in much higher concentration than in methanol-free biodiesel. Accordingly, FAME uptake dramatically decreased in all cases (compare with Figure 8).

Unfortunately, using all sorts of adsorbents with concentration as high as $2.5 \mathrm{wt} \%$ could not decrease the glycerol content from around $0.22 \mathrm{wt} \%$ in crude biodiesel to the target ASTM limit (0.02 wt \%). In such conditions, higher adsorbent loadings or a multi-step separation process should be applied to reach the standard level of free glycerol in the final product. Implementing either ways could lead to a significant FAME uptake by adsorbents, especially in mesoporous silica particles. As shown above with methanol-free biodiesel purification, the loss in 
purification yield is becoming more pronounced at lower glycerol concentration, which would be reached in multi-step purification processes.

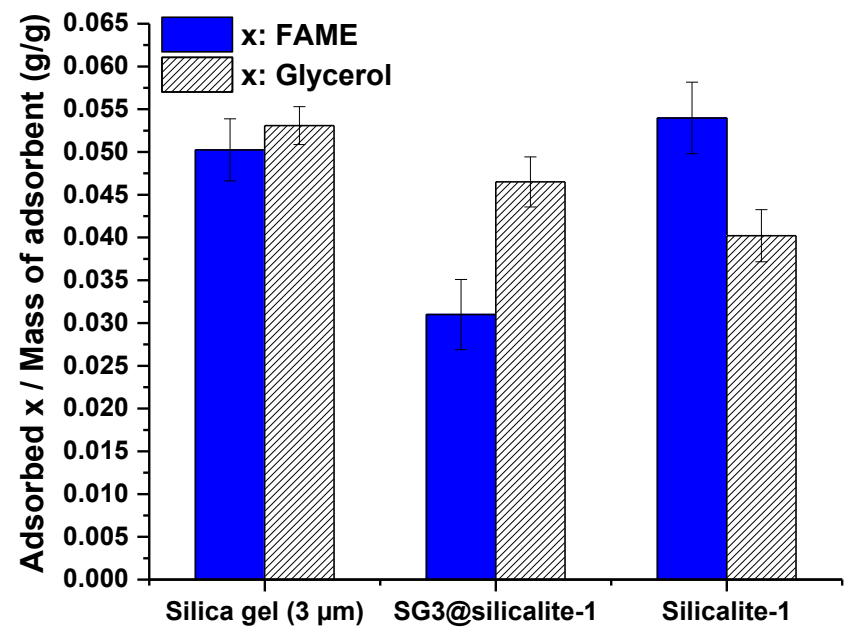

Figure 11. Glycerol and FAME adsorption using different adsorbents from methanol-containing biodiesel at room temperature (glycerol initial concentration in biodiesel $=0.22 \mathrm{wt} \%$, methanol content $\sim 0.7 \mathrm{wt} \%$, adsorbent concentration $=2.5 \mathrm{wt} \%$ ).

Glycerol adsorption from methanol-containing biodiesel at elevated temperature

The purification tests were repeated at elevated temperature using methanol-containing crude biodiesel, prompted by the clear improvement in glycerol adsorption by core@shell material from methanol-free biodiesel. In contrast to purification at $25^{\circ} \mathrm{C}$, adsorption at $100^{\circ} \mathrm{C}$ from methanol-containing biodiesel allowed to reach glycerol levels well-below standard limit (ASTM D6584) for both adsorbents (Figure 12). Manuale et al ${ }^{[25]}$ also reported on the effect of temperature increase on glycerol adsorption under vacuum.. Figure 12 shows the significant increase of equilibrium glycerol loadings for both adsorbents, SG3 and SG3@silicalite-1 upon heating at $100{ }^{\circ} \mathrm{C}$. In order to study the influence of temperature on glycerol content for a methanolcontaining biodiesel, the sample was kept at $100{ }^{\circ} \mathrm{C}$ for $1 \mathrm{~h}$ without any adsorbents which led to a significant reduction in glycerol concentration of the bulk from $0.22 \mathrm{wt} \%$ to around 0.07 $w t \%$. This diminution can be attributed to the simultaneous evaporation of volatile components, such as methanol, which were solubilising the main part of free glycerol in the mixture. In the presence of adsorbents, this precipitated glycerol comes into contact with the hydrophilic silica surface and gets adsorbed readily. In contrast, at room temperature, this fraction of glycerol is less prone to be adsorbed on the surface due to the strong interaction between glycerol and methanol.

Interestingly, these two adsorbents showed completely different behavior toward FAME, as revealed in Figure 13. A very low amount of FAME is taken-up upon heating for the core@shell material, while glycerol adsorption was significantly improved. It is believed that free-glycerol in higher concentration occupied the majority of adsorption sites at the early stages of the process. This leaves no room for bulky FAME molecules to diffuse through the microporous silicalite-1 barrier. In contrast, FAME adsorption by silica gel significantly increased. As previously mentioned, methanol at $25{ }^{\circ} \mathrm{C}$ was adsorbed in large quantity on the silica surface along with glycerol. Upon methanol evaporation at $100{ }^{\circ} \mathrm{C}$, this portion of silica gel surface was available for the bulkier molecules. As the large pore size of silica gel does not impose any diffusion restriction, FAME molecules can freely penetrate through the pores along with glycerol. However, FAME uptake from methanol-containing biodiesel was still lower than that from methanol-free biodiesel which is due to the difference in the initial glycerol concentration.

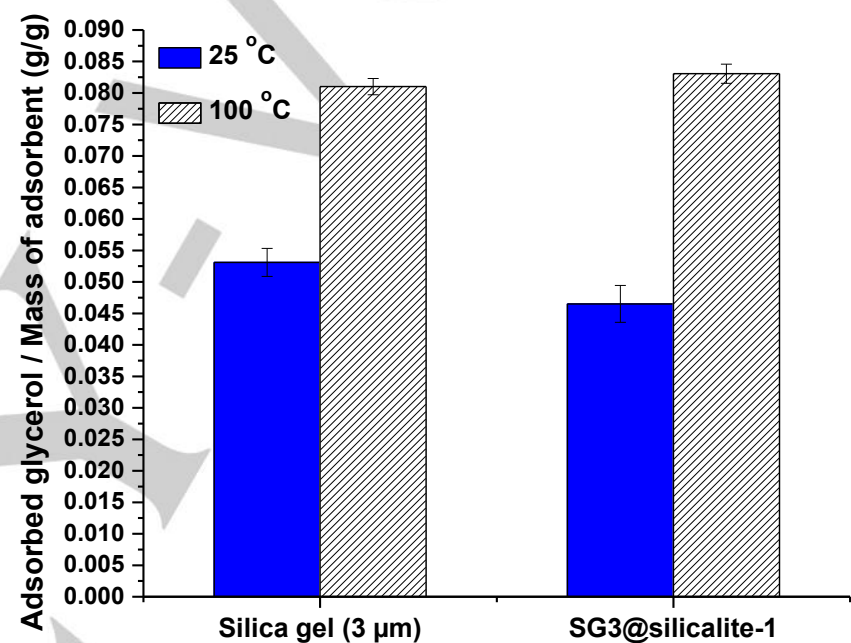

Figure 12. Adsorption of glycerol from methanol-containing biodiesel at different temperatures (glycerol initial concentration in biodiesel $=0.22 \mathrm{wt} \%$, methanol content $\sim 0.7 \mathrm{wt} \%$, adsorbent concentration $=2.5 \mathrm{wt} \%$ ).

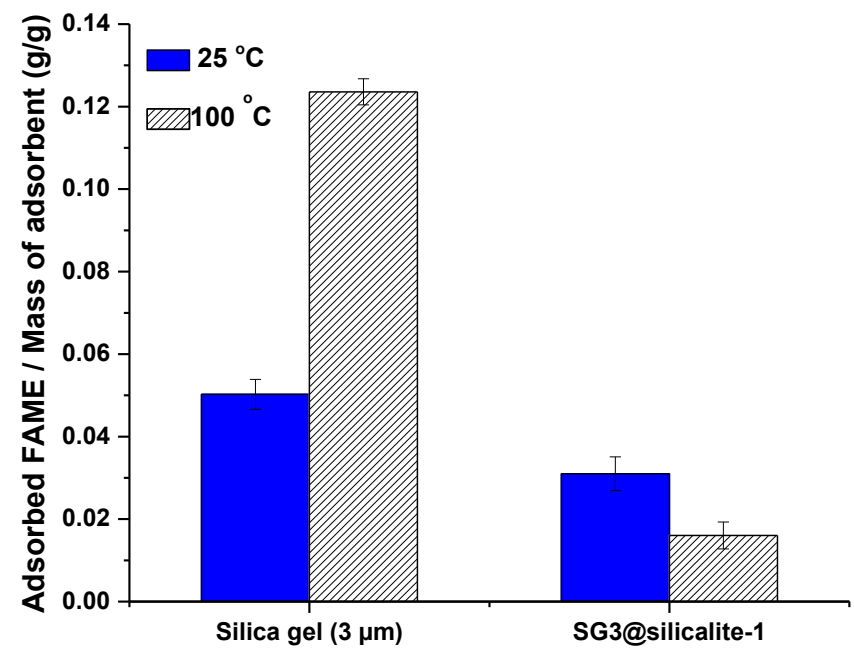

Figure 13. Uptake of FAME from methanol-containing biodiesel at different temperatures (glycerol initial concentration in biodiesel $=0.22 \mathrm{wt} \%$, methanol content $\sim 0.7 \mathrm{wt} \%$, adsorbent concentration $=2.5 \mathrm{wt} \%$ ). 


\section{Conclusions}

A new adsorbent for selective removal of free glycerol from crude biodiesel mixture was introduced, consisting of a mesoporous silica core and a microporous silicalite- 1 shell. More than $97 \%$ of the shell coverage was achieved by performing two successive secondary growth steps without any damage to the mesopore silica core. Using core particles with different sizes and pore texture, parameters such as shell thickness and the micropore-to-mesopore volume ratio can be adjusted. Glycerol adsorption tests revealed that, in addition to a high surface area and large pore volume, effective adsorbents should also possess highly size-selective pore entrance so as to maintain a larger number of adsorption sites for small molecules like glycerol and methanol. Conventional adsorbents, e.g., mesoporous silica gel, showed high FAME uptake along with glycerol adsorption, which resulted in poorer purification yield. The synthesized core@shell composite adsorbent is proven to be a suitable alternative to address the current problems of the existing sorbents in terms of glycerol removal and purification yield. A minimized effective adsorption length owing to the shortened micropores on the shell-side along with possessing the sieving ability of the parent zeolite, coupled to the core mesoporosity, supply new materials with extra ability to capture more selectively glycerol from crude biodiesel.

\section{Experimental Section}

\section{Biodiesel Preparation}

The biodiesel was prepared using the procedure presented by Alves et al. [26] via alkali-catalyzed transesterification reaction, using commercial refined corn oil, purchased from a local store, and methanol (certified ACS reagent grade, Fisher Scientific) as reactants and potassium hydroxide ( $\mathrm{KOH}$ - Sigma-Aldrich) as a catalyst. The transesterification reaction was carried out in a $500 \mathrm{ml}$ round bottom flask with a reflux condenser to prevent methanol loss, and a magnetic stirrer to keep the reaction under constant agitation. The flask was placed in an oil bath to control the reaction temperature at $65{ }^{\circ} \mathrm{C}$. The corn oil was preheated prior to adding the catalyst $\mathrm{KOH}$ pellet and methanol. The catalyst was previously dissolved into methanol until complete dissolution. This solution was also preheated at $50{ }^{\circ} \mathrm{C}$. Oil to methanol molar ratio was (1:6); the reaction time was $1 \mathrm{~h}$ and the amount of catalyst in relation to the oil mass was $0.75 \mathrm{wt} \%$. This mixture was then placed on a sealed separatory funnel and allowed to settle for at least $24 \mathrm{~h}$, and the bottom glycerol-rich layer was removed. The produced biodiesel was used as methanol-containing crude biodiesel for adsorption tests. For methanolfree tests, the methanol was completely evaporated in a rotary evaporator under $50 \mathrm{mmHg}$ vacuum at $60^{\circ} \mathrm{C}$ for $15 \mathrm{~min}$. Simple weighing method was used to determine methanol content of the methanolcontaining crude biodiesel by recording the weights of given amounts of crude biodiesel prior to and after evaporation. The sample weights remained constant after $15 \mathrm{~min}$ of evaporation under vacuum at $60^{\circ} \mathrm{C}$

\section{Synthesis of the Core@Shell Adsorbents}

The core@shell adsorbent was synthesized by adopting the seeded growth method reported in the literature. ${ }^{[5 d, 8 c]}$ After preparing the suspension of discrete silicalite-1 nanocrystals and mesoporous silica spheres, a multistep coating technique was used to produce nanozeolites coated cores, followed by a hydrothermal treatment to translate these coated cores into core@shell with a stable and uniform zeolitic shell (Scheme 1).

\section{Preparation of Silicalite-1 Nanocrystals}

The silicalite-1 nanocrystals were synthesized according to a modified procedure reported by Schoeman et al. ${ }^{[27]}$. For a typical synthesis of silicalite-1 nanocrystals with a size of approximately $70 \mathrm{~nm}, 20 \mathrm{~g}$ tetraethyl orthosilicate (TEOS) (reagent grade, 98\%-Sigma-Aldrich), as silica source, was added into $40 \mathrm{~g}$ tetrapropylammonium hydroxide (TPAOH) solution (1M in $\mathrm{H}_{2} \mathrm{O}$-Sigma-Aldrich) under continuous stirring. By adding certain amount of water, the molar composition of the synthesis mixture was adjusted to $9 \mathrm{TPAOH}: 25 \mathrm{SiO}_{2}: 480 \mathrm{H}_{2} \mathrm{O}: 100$ $\mathrm{C}_{2} \mathrm{H}_{5} \mathrm{OH}$. After prehydrolysis by stirring at room temperature for $12 \mathrm{~h}$, the clear gel was hydrothermally treated in a tightly closed propylene bottle at $80^{\circ} \mathrm{C}$ for $72 \mathrm{~h}$ until the solution turned slightly turbid. After cooling, the obtained nanocrystals were thoroughly washed with water and recovered using high speed centrifugation. After a series of centrifugation and resuspending in water, the nanocrystals were finally redispersed in distilled water to produce $0.5 \mathrm{wt} \%$ suspension. The $\mathrm{pH}$ of the suspensions was adjusted to 9-10 with ammonia solution, as suggested by Valtchev and Mintova. ${ }^{[5 b]}$

Preparation of Core Materials

Mesoporous silica spheres in three different sizes (Hexagonal Mesoporous Silica (HMS) spheres: $1.5 \mu \mathrm{m}$ and commercial silica gel spheres 3, 20-45 $\mu \mathrm{m}$ ) are used as core materials. Mono-dispersed HMS spheres were synthesized according to the literature. ${ }^{[2 f, 28]}$ In a typical producer, $2.08 \mathrm{~g}$ of hexadecylamine (technical grade, 90\%-SigmaAldrich) as mesoporogen, $180 \mathrm{ml}$ of distilled water and $200 \mathrm{ml}$ of 2propanol (fisher scientific) as solvents and $3.2 \mathrm{ml}$ of $\mathrm{NH}_{3}-\mathrm{H}_{2} \mathrm{O}(28 \%)$ as a base catalyst were mixed together until a homogenous solution was formed. $12 \mathrm{ml}$ of TEOS as silica source was added and the final mixture stirred for another $1 \mathrm{~min}$ before aging overnight at room temperature. The product has been recovered through filtration and washing with water Removing organic templates from the pores, the as-synthesized material was heated in air at $550^{\circ} \mathrm{Cfor} 6 \mathrm{~h}$. Commercial silica gel spheres with two different particle sizes (SG20: 20-45 $\mu \mathrm{m}$ and SG3: $3 \mu \mathrm{m}$ ) were purchased from SiliCycle $\AA$ Inc and used as received without further treatment.

\section{Preparation of Core@Shell Products}

Around $0.2 \mathrm{~g}$ of mesoporous silica spheres were dispersed in $5 \mathrm{ml}$ distilled water, followed by reversing their negative surface charge upon treating with a $5 \mathrm{ml}$ of $0.5 \mathrm{wt} \%$ aqueous solution of cationic low molecular weight poly(diallyldimethylammonium chloride) (PDADMAC) solution (20 wt.\% in $\mathrm{H}_{2} \mathrm{O}$-Sigma-Aldrich) under $20 \mathrm{~min}$ of stirring. Afterward, $5 \mathrm{ml}$ of negative charge silicalite- 1 nanocrystals suspension were added to be adsorbed on the surface of the cores due to electrostatic interaction. The excess nanocrystals were washed away by performing several cycles of centrifugation and redispersion of coated particles using a dilute $\mathrm{NH}_{3}-\mathrm{H}_{2} \mathrm{O}$ solution $(\mathrm{pH}$ 9.5). This intermediate product was dried in an oven at $80{ }^{\circ} \mathrm{C}$ overnight and then calcined in air at $550{ }^{\circ} \mathrm{C}$ for $5 \mathrm{~h}$, ensuring firm adherence of nanocrystals on the surface of the core material. The clear gel for secondary growth step was prepared according to Bouizi et al ${ }^{[5 \mathrm{~d}]}$ with the molar composition of 3 TPAOH: $25 \mathrm{SiO}_{2}: 1500 \mathrm{H}_{2} \mathrm{O}: 100 \mathrm{C}_{2} \mathrm{H}_{5} \mathrm{O}$. The coated core particles were dispersed in $10 \mathrm{~g}$ of clear gel by stirring at room temperature for $15 \mathrm{~min}$, followed by hydrothermal treatment at $200{ }^{\circ} \mathrm{C}$ for $45 \mathrm{~min}$ in a Par teflonlined autoclave to grow the silicalite- 1 nanocrystals adsorbed on the core 
surfaces. After cooling down the autoclave at ambient temperature and washing the product for four times with distilled water, the secondary growth step was repeated one more time to reach desirable shell coverage and uniformity. After cooling, the product was treated with a dilute $\mathrm{NH}_{3}-\mathrm{H}_{2} \mathrm{O}$ solution ( $\mathrm{pH}$ 9.5) using ultrasonic bath for $10 \mathrm{~min}$ to remove the loosely attached silicalite- 1 crystals, rinsed repeatedly with distilled water and dried at $100{ }^{\circ} \mathrm{C}$ overnight. The as-synthesized material was calcined at $550{ }^{\circ} \mathrm{C}$ for $6 \mathrm{~h}$ in air after reaching this temperature at a rate of $1 \mathrm{C} / \mathrm{min}$ to remove the TPA ${ }^{+}$template from the zeolite pores.

In order to compare the performance of final material in biodiesel purification, different types of conventional zeolites were also prepared including large microcrystals of pure silica beta zeolite (Si-BEA) using the fluoride route ${ }^{[5 \mathrm{~d}]}, \mathrm{Al}-\mathrm{ZSM}-5(\mathrm{H})$ microcrystals and sub-micron silicalite-1 crystals, both synthesized according to reported synthesis routes. ${ }^{[29]}$

\section{Material Characterization}

All products, i.e. core materials, nanocrystals, zeolites and core@shell materials, are characterized using typical characterization techniques. Powder X-ray diffraction patterns of all samples were recorded using a Siemens powder diffractometer $(40 \mathrm{kV}, 40 \mathrm{~mA})$ with $\mathrm{CuK}_{\alpha}$ radiation $(\lambda=$ $1.54059 \mathrm{~A})$. The transmission electron microscopy (TEM) images were recorded using a JEOL JEM 1230 electron microscope after dispersing samples in methanol and depositing on carbon-coated nickel grids. Scanning electron microscopy (SEM) studies were performed using a JEOL JSM-840A scanning electron microscope. To prepare the sample, a small quantity of the powder sample was placed onto the SEM sample holder and then coated two times with gold and palladium. Simultaneous thermogravimetry and differential scanning calorimetery (TGA-DSC) measurements were performed using a Netzsch STA 449C thermogravimetric analyzer. Nitrogen adsorption-desorption isotherms were measured at liquid nitrogen temperature $\left(-196{ }^{\circ} \mathrm{C}\right)$, using a Quantachrome Autosorb-1 adsorption analyzer. Prior to the measurements, the samples were evacuated at $200{ }^{\circ} \mathrm{C}$ for at least $12 \mathrm{~h}$, under the vacuum, provided by a turbomolecular pump. The linear part of Brunauer-Emmett- Teller (BET) equation was used to calculate the specific surface area from adsorption data obtained which mainly occurred at $P / P_{0}$ between 0.05 and 0.2 . Total pore volume of micropores and mesopores was estimated from the amount of nitrogen adsorbed at $\mathrm{P} / \mathrm{P}_{0}=0.95$. For advanced porosity analysis, cumulative pore volumes and pore size distributions were determined by using non-local density functional theory (NLDFT) method applying the NLDFT metastable adsorption branch kernel and considering sorption of nitrogen at $-196^{\circ} \mathrm{C}$ in silica as a model adsorbent and cylindrical pores as a pore model. Micropore volumes of the zeolites and core@shell materials, as well as their pore size distributions, were determined using NLDFT methods. The Quantachrome Autosorb-1 1.55 software was used for data interpretation.

\section{Glycerol Adsorption Test}

Crude biodiesel (with and without methanol) was purified using different types of adsorbents; mesoporous silica spheres, zeolites and core@shell particles. Prior to use, all adsorbents were dried at $150{ }^{\circ} \mathrm{C}$ in a vacuum oven overnight to eliminate the free moisture. Approximately, 20-30 mg of the dry adsorbent was introduced into a $5 \mathrm{ml}$ glass vial containing $1 \mathrm{~g}$ crude biodiesel with a given initial glycerol concentration $\left(w_{0}\right)$, i.e. $w_{0}=$ $0.065 \mathrm{wt} \%$ for methanol-free biodiesel and $w_{0}=0.22 \mathrm{wt} \%$ for methanolcontaining biodiesel. Average methanol content of the methanolcontaining biodiesel samples was measured as $0.7 \mathrm{wt} \%$ right before performing the adsorption tests. Freshly prepared biodiesels were used in all tests since the glycerol concentration were found to change over time when stored due to gradual evaporation of methanol. Samples were equilibrated for $12 \mathrm{~h}$ at $25^{\circ} \mathrm{C}$ in an incubator while being stirred with a magnetic stirrer. Studying the temperature effect on the adsorption, all experiments were repeated at $100^{\circ} \mathrm{C}$ in an oil bath. Upon equilibration, the adsorbents were separated from the purified biodiesel by sedimentation/centrifugation. Glycerol concentrations in purified supernatant were measured after equilibration via GC-FID technique in order to quantitatively determine the initial and equilibrium concentrations in biodiesel and used to calculate the equilibrium adsorption capacity $\left(\mathrm{q}_{\mathrm{e}}\right)$ of dry adsorbent using following material balance equation.

$$
q_{e}=\frac{\left(w_{0}-w_{e}\right) \times m_{\text {biodiesel }}}{m_{\text {adsorbent }}} \times \frac{1}{100}
$$

where $q_{e}=$ amount of glycerol adsorbed by the adsorbent $(g / g), w_{0}=$ initial mass percent of glycerol $(\mathrm{g} / \mathrm{g}), \mathrm{w}_{\mathrm{e}}=$ mass percent of glycerol at equilibrium $(\mathrm{g} / \mathrm{g}), \mathrm{m}_{\text {biodiesel }}=$ initial mass of biodiesel $(\mathrm{g})$, and $\mathrm{m}_{\text {adsorbent }}=$ mass of dry adsorbent $(\mathrm{g})$.

In order to determine the uptake of bulkier molecules, such as FAME, by the adsorbents, thermogravimetric experiments were performed on the used adsorbents. In a typical procedure, following the recovery of adsorbent after adsorption, the used adsorbents were quickly washed with hexane to remove the bulk molecules, then the volatile compounds were evaporated at $35^{\circ} \mathrm{C}$ for $24 \mathrm{~h}$. Around $2 \mathrm{mg}$ of dried adsorbent were placed in the alumina crucible (with no lid) of a TGA-DSC instrument and heated from $35^{\circ} \mathrm{C}$ to $700^{\circ} \mathrm{C}$ in air flow $(20 \mathrm{ml} / \mathrm{min})$ at a heating rate of 10 ${ }^{\circ} \mathrm{C} / \mathrm{min}$. The measured mass loss at high temperatures $\left(150-550{ }^{\circ} \mathrm{C}\right)$ was considered as the mass of non-volatile organic molecules which were trapped/adsorbed inside the pores of adsorbents. The measured nonvolatile organic content was then translated to the bulkier molecules uptake by the adsorbent upon subtracting the mass of adsorbed glycerol measured by GC-FID. The standard uncertainty for glycerol and FAME uptakes were calculated/estimated following the guideline provided in GUM (Guide to the expression of uncertainty in measurement ${ }^{[30]}$ ). The corresponding expanded uncertainties are presented with each data set using a coverage factor $\mathrm{k}=2$, providing a level of confidence of approximately $95 \%$.

\section{Analytical Method}

Free glycerol in biodiesel was determined using an off-line gas chromatography, Thermo Scientific TRACE GC Ultra, equipped with a flame ionization detector (FID), automated by the TriPlus liquid autosampler. The analytical column was a non-polar DB-5MS (30 m, $0.25 \mathrm{~mm}$ ID, $0.25 \mu \mathrm{m}$ film) from Agilent technologies, Inc. Calibration was achieved by the use of 1 wt\% 1,4-butanediol (Sigma-Aldrich) in Pyridine (Alfa-Aesar, 99+\%) as an internal standard for glycerol (ultra pure, MP Biochemicals). Transforming polar and high boiling glycerol and internal standard into more volatile silylated derivatives, they were derivatized using N,O-Bis(trimethylsilyl)trifluoroacetamide (BSTFA $\geq 99.0 \%$, Fulka) derivatization reagent and pyridine as a solvent and silylation catalyst. Five calibration solutions were prepared containing glycerol and internal standard at concentrations specified in the ASTM D6584 method. $200 \mu \mathrm{L}$ of the derivatization agent, BSTFA, were added to each calibration solution in a vial. For each biodiesel sample, approximately $100 \mathrm{mg}$ of homogenized sample were accurately weighed $( \pm 0.1 \mathrm{mg})$ in a vial; then $80 \mu \mathrm{L}$ of internal standard solution and $200 \mu \mathrm{L}$ of BSTFA were added. All vials were sealed and shaken vigorously for $20 \mathrm{~min}$ and then aged at $60^{\circ} \mathrm{C}$ for $20 \mathrm{~min}$. Finally, all mixtures were dissolved in $n$-heptane (ACS grade, $\mathrm{BDH}$ ). For analysis, $1 \mu \mathrm{L}$ of the reaction mixture was automatically injected into the GC instrument at an oven temperature of $60^{\circ} \mathrm{C}$. After an isothermal period of $1 \mathrm{~min}$, the oven was heated at $15^{\circ} \mathrm{C} / \mathrm{min}$ to $230^{\circ} \mathrm{C}$ and then to $300^{\circ} \mathrm{C}$ at $50^{\circ} \mathrm{C} / \mathrm{min}$ (held for $5 \mathrm{~min}$ ). Nitrogen was used as the carrier gas at a flow rate of $1 \mathrm{ml} / \mathrm{min}$. The detector temperature was set 
to $350^{\circ} \mathrm{C}$. The amount of glycerol in each sample was calculated using the calibration function derived from the glycerol calibration curve.

\section{Acknowledgements}

The authors acknowledge the financial support from the National Science and Engineering Research Council (Canada) and the Fonds québécois de la recherche sur la nature et les technologies (FRQNT).

Keywords: Biodiesel - Free glycerol $•$ Adsorption - Purification • Core@shell

[1] a) R. Ghosh Chaudhuri, S. Paria, Chem. Rev. 2011, 112, 2373-2433; b) H. Wang, L. Chen, Y. Feng, H. Chen, Acc. Chem. Res. 2013, 46, 16361646.

[2] a) V. Valtchev, Chem. Mater. 2002, 14, 4371-4377; b) V. Valtchev, Chem. Mater. 2002, 14, 956-958; c) W. Yang, X. Wang, Y. Tang, Y. Wang, C. Ke, S. Fu, J. Macromol. Sci., Part A: Pure Appl.Chem. 2002 39, 509 - 526; d) X. D. Wang, W. L. Yang, Y. Tang, Y. J. Wang, S. K. Fu, Z. Gao, Chem. Commun. 2000, 2161-2162; e) A. Dong, Y. Wang, D. Wang, W. Yang, Y. Zhang, N. Ren, Z. Gao, Y. Tang, Microporous Mesoporous Mater. 2003, 64, 69-81; f) A. Dong, N. Ren, W. Yang, Y. Wang, Y. Zhang, D. Wang, J. Hu, Z. Gao, Y. Tang, Adv. Funct. Mater 2003, 13, 943-948.

[3] a) R. Kanthasamy, K. Barquist, S. C. Larsen, Microporous Mesoporous Mater. 2008, 113, 554-561; b) W. Song, R. Kanthasamy, V. H. Grassian, S. C. Larsen, Chem. Commun. 2004, 1920-1921; c) J. C. Groen, T. Bach, U. Ziese, A. M. Paulaime-van Donk, K. P. de Jong, J. A. Moulijn, J. Pérez-Ramírez, J. Am. Chem. Soc. 2005, 127, 10792 10793; d) J. C. Groen, J. C. Jansen, J. A. Moulijn, J. Pérez-Ramírez, J. Phys. Chem. B 2004, 108, 13062-13065.

[4] a) Y. Deng, C. Deng, D. Qi, C. Liu, J. Liu, X. Zhang, D. Zhao, Adv. Mater. 2009, 21, 1377-1382; b) J. Zheng, Q. Zeng, J. Ma, X. Zhang, W. Sun, R. Li, Chem. Lett. 2010, 39, 330-331.

[5] a) J. Zheng, Q. Zeng, Y. Zhang, Y. Wang, J. Ma, X. Zhang, W. Sun, R. Li, Chem. Mater. 2010, 22, 6065-6074; b) V. Valtchev, S. Mintova, Microporous Mesoporous Mater. 2001, 43, 41-49; c) Y. Bouizi, G. Majano, S. Mintova, V. Valtchev, J. Phys. Chem. C 2007, 111, 45354542; d) Y. Bouizi, I. Diaz, L. Rouleau, V. Valtchev, Adv. Funct. Mater 2005, 15, 1955-1960; e) Y. Bouizi, L. Rouleau, V. P. Valtchev, Chem. Mater.2006, 18, 4959-4966.

[6] F. Caruso, R. A. Caruso, H. Möhwald, Science 1998, 282, 1111-1114.

[7] a) J. Zhang, T. Zhang, X. Zhang, W. Liu, H. Liu, J. Qiu, K. L. Yeung, Catal. Today 2014, 236, 34-40; b) Y. Yoneyama, X. San, T. Iwai, N. Tsubaki, Energy Fuels 2008, 22, 2873-2876; c) N. Jiang, G. Yang, X Zhang, L. Wang, C. Shi, N. Tsubaki, Catal. Commun. 2011, 12, 951954; d) X. Wang, Y. Cui, Y. Wang, X. Song, J. Yu, Inorg. Chem. 2013, 52, 10708-10710; e) J. Wang, D.-M. Do, G.-K. Chuah, S. Jaenicke, ChemCatChem 2013, 5, 247-254; f) E. A. Khan, A. Rajendran, Z. Lai, Ind. Eng. Chem. Res. 2010, 49, 12423-12428.

[8] a) C. Casado, J. Bosque, N. Navascués, C. Téllez, J. Coronas, Microporous Mesoporous Mater. 2009, 120, 69-75; b) E. A. Khan, A
Rajendran, Z. Lai, Chem. Eng. Res. Bull. 2013, 16, 1-15; c) G. D. Pirngruber, C. Laroche, M. Maricar-Pichon, L. Rouleau, Y. Bouizi, V Valtchev, Microporous Mesoporous Mater. 2013, 169, 212-217.

[9] A. Carrero, Á. Pérez, in Advances in Biodiesel Production (Eds.: R. Luque, J. A. Melero), Woodhead Publishing, 2012, pp. 91-130.

[10] I. J. Stojković, O. S. Stamenković, D. S. Povrenović, V. B. Veljković, Renewable Sustainable Energy Rev. 2014, 32, 1-15.

[11] M. Berrios, R. L. Skelton, Chem. Eng. J. 2008, 144, 459-465.

[12] a) S. L. Dmytryshyn, A. K. Dalai, S. T. Chaudhari, H. K. Mishra, M. J. Reaney, Bioresour. Technol. 2004, 92, 55-64; b) V. A. Mazzieri, C. R. Vera, J. C. Yori, Energy Fuels 2008, 22, 4281-4284.

[13] J. C. Yori, S. A. D'Ippolito, C. L. Pieck, C. R. Vera, Energy Fuels 2006 , 21, 347-353

[14] a) S. Özgül-Yücel, S. Türkay, J. Am. Oil Chem. Soc. 2003, 80, 373-376; b) Z. J. Predojević, Fuel 2008, 87, 3522-3528; c) A. L. M. T. Pighinelli, R. A. Ferrari, A. M. R. O. Miguelb, K. J. Parka, Grasas Aceites 2011, 62, 171-180.

[15] K. Mis Solval, S. Sathivel, J. Am. Oil Chem. Soc. 2012, 89, 1713-1721.

[16] E. A. Khan, E. Hu, Z. Lai, Microporous Mesoporous Mater. 2009, 118, 210-217.

[17] a) M. Thommes, Chem. Ing. Tech. 2010, 82, 1059-1073; b) C. J. Rasmussen, A. Vishnyakov, M. Thommes, B. M. Smarsly, F. Kleitz, A V. Neimark, Langmuir 2010, 26, 10147-10157

[18] http://www.iza-structure.org/databases/, 2014

[19] M. B. Carlos Vera, Juan Yori, Gerardo Torres, Debora Manuale, Sergio Canavese and Jorge Sepúlveda in Biodiesel - Feedstocks and Processing Technologies, (Eds.:M. Stoytcheva), InTech, 2011, pp. 427458.

[20] D. P. Serrano, R. Van Grieken, P. Sánchez, R. Sanz, L. Rodríguez, Microporous Mesoporous Mater. 2001, 46, 35-46.

[21] P. Chand, C. V. Reddy, J. G. Verkade, T. Wang, D. Grewell, Energy Fuels 2009, 23, 989-992.

[22] G. Üstün, J. Am. Oil Chem. Soc. 1996, 73, 203-210.

[23] J. Xiao, J. Wei, Chem. Eng. Sci. 1992, 47, 1143-1159.

[24] a) S. Kulprathipanja, R. B. James, in Zeolites in Industrial Separation and Catalysis, (Eds.: S. Kulprathipanja), Wiley-VCH Verlag GmbH \& Co. KGaA, 2010, pp. 173-202; b) I. N. Lykakis, C. Ferreri, S. A. Grabovskiy, C. Chatgilialoglu, Tetrahedron 2010, 66, 2203-2209.

[25] a) D. L. Manuale, E. Greco, A. Clementz, G. C. Torres, C. R. Vera, J. C. Yori, Chem. Eng. J. 2014, 256 372-379; b) D. L. Manuale, V. M. Mazzieri, G. Torres, C. R. Vera, J. C. Yori, Fuel 2011, 90, 1188-1196.

[26] M. J. Alves, S. M. Nascimento, I. G. Pereira, M. I. Martins, V. L. Cardoso, M. Reis, Renewable Energy 2013, 58, 15-20.

[27] B. J. Schoeman, K. Higberg, J. Sterte, Nanostruct. Mater. 1999, 12, 4954.

[28] M. Grün, G. Büchel, D. Kumar, K. Schumacher, B. Bidlingmaier, K. K Unger, in Studies in Surface Science and Catalysis, Vol. 128 (Eds.: G. K. K.K. Unger, J. P. Baselt), Elsevier, 2000, pp. 155-165.

[29] H. Robson, Editor, Verified Synthesis of Zeolitic Materials, Second Edition, Elsevier, 2001.

[30] Evaluation of measurement data- Guide to the expression of uncertainty in measurement, JCGM 100:2008 
Entry for the Table of Contents (Please choose one layout)

Layout 1:

\section{FULL PAPER}

A new adsorbent for selective removal of free glycerol from crude biodiesel mixture is introduced, consisting of mesoporous silica core with uniform microporous silicalite-1 shell. Glycerol sorption revealed that, effective adsorbents possess highly size-selective pore entrance so as to maintain a larger number of adsorption sites for small molecules (e.g., glycerol, methanol)

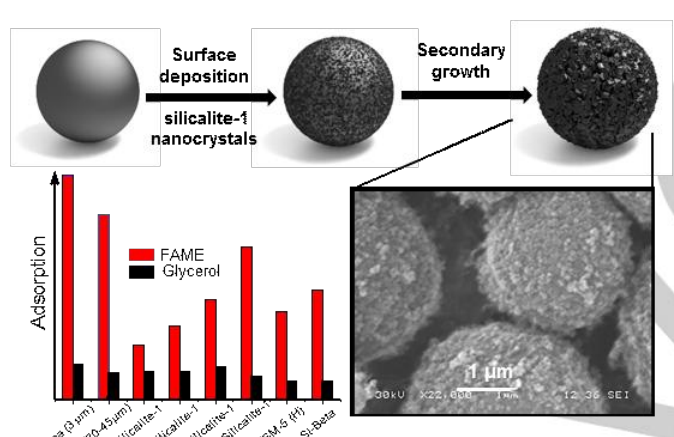

Nima Masoumifard, Pablo M. Arnal, Serge Kaliaguine* and Freddy Kleitz*

Page No. - Page No.

Title

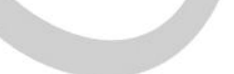

Layout 2:

\section{FULL PAPER}

((Insert TOC Graphic here; max. width: $11.5 \mathrm{~cm}$; max. height: $2.5 \mathrm{~cm})$ )
Author(s), Corresponding Author(s)*

Page No. - Page No.

Title

Text for Table of Contents 
Click here to download Supporting Information: Masoumifard_Kaliaguine_Kleitz_CSC_SI.docx

Supporting Information

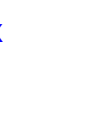

(1)

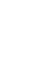

$\sqrt{2}$

(1)

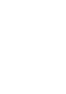

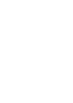

更

(1)

(1)

.

.

.

.

.

.

.

.

.

.

.

.

.

.

.

.

.

.

.

.

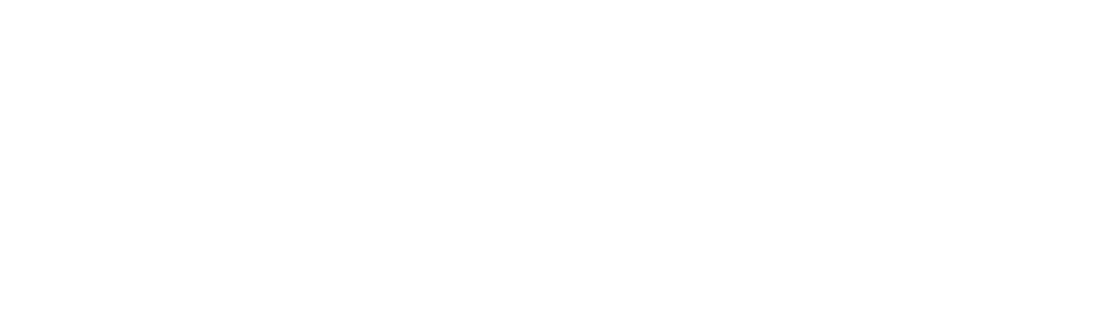

